\title{
Managing the Pharmacy Benefit: The Formulary System
}

\author{
Robert B. Goldberg
}

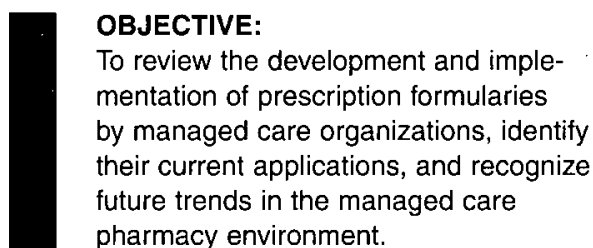

OBJECTIVE:

To review the development and implementation of prescription formularies managed care organizations, identify future trends in the managed care pharmacy environment.

\author{
DATA SOURCES: \\ Current journal articles and texts regard- \\ ing the use of formularies and the man- \\ aged care environment. \\ DATA SYNTHESIS: \\ Not applicable. \\ CONCLUSION: \\ Formulary systems have proven to be a
}

\begin{abstract}
valuable means to control the pharmacy benefit and can be expected to expand in both scope and sophistication.
\end{abstract}

\section{KEYWORDS:}

Formulary, PBM, P\&T committee

J Managed Care Pharm 1997; 3: 565-573
In 1960 public and private expenditures on health care amounted to $\$ 26.8$ billion; by 1994 this amount had grown to $\$ 949.4$ billion. Spending for pharmaceuticals accounted for $8.2 \%$ of this total. ${ }^{1}$ This rapid inflation in medical spending, relative to the general consumer inflation rate, caused a paradigm shift in healthcare insurance from fee-for-service reimbursement to a managed care environment. In 1976 there were six million people enrolled in HMOs. By 1995 that number had reached 58.2 million. ${ }^{.}$This surge of enrollment in managed care organizations (MCOs) led to the increased use of outpatient medication formularies that are now such an integral component of the managed care pharmacy landscape.

This article describes the beginnings of the formulary system as a tool used by hospitals in the 1950s, to its current application in the outpatient setting. It also discusses the types, impact, and effectiveness of the various levels of formulary management. In addition, the article examines the future structure that prescription formularies are likely to attain and the forces that will drive tomorrow's prescription benefit.

\section{DEVELOPMENT OF THE FORMULARY SYSTEM}

The prescription formulary was developed initially as a management tool. Early on, hospitals realized the need for an inpatient formulary. Without a listing of approved medications, there was no way a hospital pharmacy could control its inventory and ensure that physicians would have an adequate and consistent supply of medication for their day-to-day needs.

In 1950, the Joint Commission on Accreditation of Hospitals (JCAH) encouraged formation of a Pharmacy and Therapeutics (P\&T) committee and the development of a formulary system. By 1965, a P\&T committee was required by JCAH before a hospital could receive accreditation. $^{2}$

As early as 1969, the Task Force on Prescription Drugs realized that while "the use of a formulary is not a guarantee of high-quality medical care, rational prescribing, effective utilization review, and control of costs...the achievement of these objectives in a drug program is difficult, if not impossible, without it."'

\section{A Author}

Robert B. Goldberg R.Ph., Drug Therapy Management Pharmacist, Express Scripts, Inc.

AUTHOR CORRESPONDENCE: Robert B. Goldberg R.Ph., Drug Therapy Management Pharmacist, Express Scripts Inc., 14000 Riverport Drive Maryland Heights, MO 63043.

AC CE CREDIT: This is article number 233-000-97-086-H04 in AMCP's continuing education program. It affords 1.0 hour (0.1 CEU) of credit. PE. Learning objectives and test questions follow on page 576

and Copyright (O) 1997, Academy of Managed Care Pharmacy, Inc. All rights reserved. 
With the shift of health insurance toward managed care, insurers began to look at ways to control the utilization of the pharmaceutical benefit. At the time, most plans had experience with formularies for inpatient settings but not for ambulatory patients. The first approach was to have one unified formulary that would be applicable to both inpatient and outpatient care. The MCOs soon realized that the most efficacious product for an inpatient setting would not necessarily be the most appropriate therapy for outpatient use. Quite often the cost difference between pharmaceuticals purchased in the hospital and the same drugs purchased in a community pharmacy was tremendous. The cost of medication varied so widely that an inexpensive medication in the hospital could be extremely expensive when purchased at a retail pharmacy. So even though the same formulary might not have worked for both environments, cost savings had been proven in hospitals and increasing prescription drug costs made formularies a necessity for managing costs in the outpatient setting.

In addition to listing approved products, a formulary system should contain an educational component, organizational policies on prescribing, and procedures for the use of certain medications. It should also contain a drug use evaluation (DUE) process and, when appropriate, a drug utilization review (DUR) component. Finally, the system must have a procedure for prior authorization to use nonformulary medication when medically necessary.

\section{THE P\&T COMMITTEE}

A formulary is much more than a listing of drugs; it is an entire system established to optimize patient care through safe, appropriate, effective, and economic use of drugs. The cornerstone of this formulary system is the Pharmacy and Therapeutics committee. The role of the P\&T committee is to decide the amount of control and form the formulary will take as well as to design and coordinate all other aspects of the system. The $P \& T$ committee also is the communications link between the MCO's medical staff and the pharmacy providers. ${ }^{4}$

The size of the committee varies according to the needs of the managed care organization. While one study of 81 HMOs reported that the average P\&T committee size was $9.67 \mathrm{mem}$ bers, the number of members actually ranged from four to 19 members. ${ }^{5}$

Committee members usually include physicians (often from specialty areas with high prescription use such as primary care, internal medicine, pediatrics), pharmacists (both clinical and community), the plan's medical director, and sometimes an outside consultant or personnel from other departments within the MCO.

Often there are additional individuals or subcommittees that conduct research and report back to the P\&T committee. One such subcommittee may be a formulary committee comprised of physicians, pharmacists, nurses, and administrative personnel such as the plan's medical director and financial consultants. The function of this committee is to evaluate therapeutic classes of pharmaceuticals and make recommendations to the P\&T committee for inclusion in the formulary.

Some MCOs do not have their own formulary subcommittee, but instead contract with a Pharmacy Benefit Management (PBM) company for its pharmacy services. Most PBMs have their own $\mathrm{P} \& \mathrm{~T}$ committees that develop and approve a master formulary for the PBM. The P\&T committee of the MCO can then take this formulary, along with the clinical and financial research provided by the PBM, and develop its own subset or custom formulary.

Other individuals or groups that operate independently and report back to the P\&T committee might include quality assurance experts, drug information personnel, or consultants in pharmacology or pharmacoeconomics. Additional subcommittees may include education, DUR, and DUE.

\section{FORMULARY TYPES}

Any P\&T committee must first decide what type of formulary to administer. Each $\mathrm{MCO}$ will use its own terminology and definitions to describe its formulary, and for those unfamiliar with it, the terminology can be somewhat intimidating Formularies can be described as open, closed, or partially closed; restricted or incentivized; positive, negative, or preferred. In addition, the committees can mandate use of generi drugs, request substitution, and designate who can prescribe and how much.

\section{Open}

An open formulary is a relatively comprehensive list of drugs and usually contains few if any restrictions on providers. Open formularies may have preferred products that art promoted to the prescriber by the P\&T committee. Efforts to encourage prescribing of preferred products include newsletters and concurrent DUR messages recommending that pharmacists use preferred products. Since there are no restrictions if the preferred product is not dispensed, this type of formulary often has little impact on physicians and pharmacists; consequently it does very little to meet the goals of an MCO to control utilization and expenses.

\section{Closed}

A closed formulary is a limited list of drugs chosen by the P\&T committee. It typically contains 300 to 1,000 drug dosage forms. Only medications on this closed list will be cov ered by the prescription drug plan. Usually, this list consists o only brand-name medications; generics for these brand-name drugs also are covered. Closed formularies generally offer several choices in each therapeutic category. When developing a closed formulary, the $\mathrm{P} \& \mathrm{~T}$ committee must also set up protocols for obtaining authorization to use a nonformulary medication. Such authorization may require only a letter of medical necessity from the prescribing physician or it may mean 
documenting treatment failure with the formulary medication before authorization is granted. Closed systems require more effort than open systems, in that the physician must choose a product from a limited formulary and pharmacists often must contact the prescriber to adjust the prescription when the medication ordered is not on the formulary. Compared to open formularies, however, closed formularies contribute to more rational and objective pharmacotherapy, produce high rates of prescriber compliance, foster proper generic utilization, assist in driving performance- or market share-based contracts with pharmaceutical manufacturers, and may offer incentives to prescribers and pharmacists for certain interventions. ${ }^{2}$

For many years employee and union demands compelled employers to offer only health benefits with full open formularies. As costs continue to rise, however, there is more and more pressure to control prescription costs. This, coupled with the large number of medications that have gone off patent and the increased number of 'me too' drugs (offering no benefit over existing products but available at reduced cost) has led to the increased use of closed formularies, which offer the greatest amount of savings and control to the MCO. In 1995, only 8.3 percent of employers used a closed formulary design; by the end of 1997 it is forecast that 16.7 percent will offer closed formularies. ${ }^{6}$

\section{Partially Closed}

A partially closed formulary is essentially an open formulary with either a few selected drugs that are not covered, or one in which reimbursement might be denied for an entire class of drugs. Whether or not a medication is a covered benefit often depends on clinical necessity and cost. For example, this type of formulary might not cover Retin-A for patients over a certain age, or perhaps entire drug classes (such as anorexients) are not covered. Other forms of partially closed formularies are plans that will only cover one brand of a duallicensed brand-name product. Sometimes the medication has a lifetime cap, such as one course of nicotine patches. In such cases the decision is usually based on manufacturer contractual agreements. These may be tied to volume discounts or rebates, or be driven by market share targets. If only one class of drugs is affected, the impact may be felt by relatively few patients. For those patients, however, there is often no therapeutic alternative when an entire class of drugs is blocked. However, if the limitations are only placed on dual-licensed products, impact is minimal as there is always an identical alternative from a different manufacturer. Partially closing a formulary gives immediate control over utilization and can be an extremely effective method to control costs associated with expensive therapeutic classes that offer effective alternatives

\section{Restricted}

A formulary may be restricted in many ways. A common restriction is requiring that generics be used whenever possible. Generics give managed care plans and consumers the opportunity to utilize clinically equivalent products that may cost as much as $60 \%$ less than branded counterparts. ${ }^{6}$

Sometimes brand-name medications are not covered at all; in other cases branded drugs can be used if the member pays the difference in price. The impact of this type of restricted formulary is minimal because most physicians indicate on the prescription blank that substitution is permitted. Thus, the choice to use the brand product is usually a conscious decision made by the patient. The pharmacist, however, may be required to explain the benefit to the member or call the physician when a prescription is ordered "dispense as written." In most cases, rules regarding the use of generics are inflexible and authorization is not granted for the brand-name product unless the physician demonstrates true medical necessity-a fairly unlikely occurrence.

Another type of restricted formulary is one in which only specific physicians may prescribe a certain medication. These are generally very expensive medications which require a high level of expertise in prescribing and in monitoring treatment. An example would be a case where growth hormones may only be prescribed by an endocrinologist, or where certain antineoplastic agents can only be prescribed by an oncologist. The impact of this type of restrictive formulary is really minimal since these medications should only be dispensed after evaluation by a specialist authorized by the plan. In some cases, pharmacists might encounter difficulties when primary care physicians attempt to write prescriptions for restricted products that have been recommended by specialists.

\section{Incentivized}

An incentivized formulary is one that promotes the use of preferred products through economic reward. The incentive can be for the physician, the pharmacist, or the patient.

For physicians, the incentive is usually some form of risksharing arrangement between the physician and the managed care organization. When the physician is fully or partially at risk for the medication he or she prescribes, part of the capitation allowance is witheld and prescription costs are deducted from this withhold. At the end of the contract period the physician receives the money remaining in the withhold account. To encourage formulary compliance some plans only penalize the physician for use of nonformulary medications. Patient incentives take many forms. The common element is the amount of the prescription benefit that is nonreimbursable. See Figure 1 for the most commonly utilized patient incentives.

Pharmacist incentives also take a variety of forms. In one example, the MCO may increase the dispensing fee to the pharmacy when either a generic or formulary medication is dispensed. A newer trend is when the MCO, through its contracted PBM, pays a cognitive fee to the dispensing pharmacist when a prescription is changed to a formulary medication as a result of an intervention by the pharmacist at the time of dispensing.

While almost half (49\%) of HMOs did not utilize any type of pharmacist incentives in 1995, nearly one-third 
Figure 1. Methods of Placing Employees at Risk for the Pharmacy Benefit ${ }^{5}$

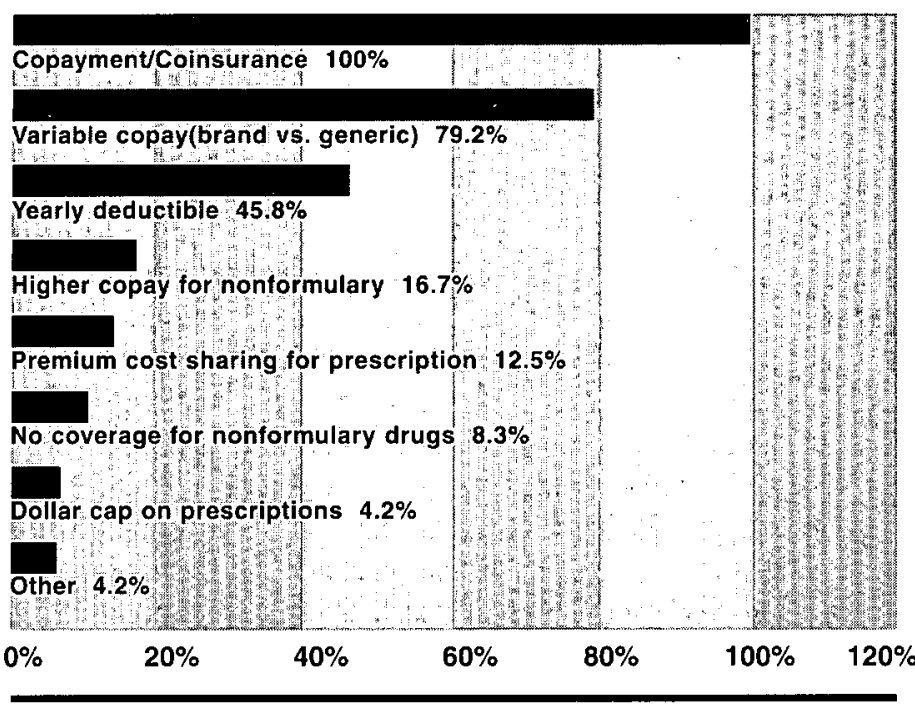

$(32.7 \%)$ did utilize differential dispensing fees for generic drugs. For formulary drugs, differential dispensing fees are expected to be utilized by $24.5 \%$ of HMOs in 1997, even though only $2 \%$ of the plans used them in 1995. Only one fifth $(20.4 \%)$ report that they do not plan to employ pharmacist incentives in 1997.6

The impact of this type of formulary depends on to whom the financial incentive is offered. The impact on the pharmacist can be substantial. While it does require time and effort on the part of the pharmacist to effect a change to the preferred medication, this is the only type of formulary that attempts to reimburse the pharmacist for his or her time and cognitive abilities. Depending on the plan design, this type of arrangement can greatly benefit someone who seeks to maximize profits. With the average prescription cost for 1997 estimated at $\$ 27.40,{ }^{6}$ even a 50 -cent increase via dispensing fees can mean an additional two percent profit.

If the patient bears the extra cost of the nonformulary medication, there may be more work for the pharmacist. Patients, however, are often aware of their own benefit situation, and request that the doctor prescribe the formulary medication at the outset.

If the physician is at risk either partially or completely he or she is more likely to ensure that only formulary medications are used. Thus, this type of arrangement tends to bring about the tightest utilization control.

Many managed care organizations depend on PBMs to integrate claims information in a manner that clearly documents physician prescribing patterns. This allows them to develop 'report cards,' detailing how a physician's prescribing habits compare to his or her peers. Such report cards are used for academic detailing to try to increase formulary compliance among doctors who are high utilizers of nonformulary medications.
This information is increasingly requested by physicians themselves once their capitation arrangement places them either partially or fully at risk for the medications they prescribe.

In the future, as more health plans place physicians at risk for the medications they prescribe, a new role for the pharmacist will likely evolve. Large group practices will conceivably employ a pharmacist for expert drug information to help manage formulary decisions.

\section{Managed}

Several types of formulary control are referred to as managed. These can effect great control over utilization, but they usually require a substantial amount of work by the MCO prior to implementation.

One such formulary manages a certain group of medications by making them available only when authorization is obtained from the plan (or the plan's PBM) before the medication is prescribed. While this can be extremely effective from a utilization control standpoint, highly-defined protocols must be established in advance that determine exactly when prior authorization will or will not be granted. The impact of this falls more on the physician than the pharmacist, because it is the former who is required to obtain authorization from the MCO. This can be a rather labor-intensive way to control utilization because there must always be enough staff on duty to evaluate requests for nonformulary medications.

Another type of managed formulary controls usage through stepped-care or the use of critical pathways. In this formulary there are defined treatment patterns based on the physician's diagnosis. Before a more expensive nonformulary medication may be utilized, a clinical trial must be attempted with the formulary medication first. If treatment fails with the formulary medication, the physician provides documentation and can then receive authorization to proceed to the next step of treatment. For example, when treating benign prostatic hypertrophy, a required course of therapy with Terazosin must be tried before Proscar would be authorized.

While this type of management is effective, it can also be labor-intensive, requiring exhaustive research and planning before implementation. Once again, the primary impact is on the physician who must demonstrate the need for a nonformulary medication to the managed care plan prior to prescribing the medication.

\section{Positive and Negative}

The manner in which the formulary is presented to pharmacists and physicians is sometimes referred to as positive or negative. A positive formulary is essentially a closed formulary listing all the products that can be prescribed as covered benefits. If a medication is not included in the list, it is not a covered benefit and may only be dispensed after medical necessity is demonstrated and prior authorization has been obtained. A negative formulary is generally a partially closed formulary and, rather than listing every medication that is covered, the 
published formulary includes only those medications for which the plan will not reimburse.

\section{Therapeutic Intervention}

An additional method to encourage adherence to a plan's formulary is by means of therapeutic intervention. In this system the P\&T committee develops a list of preferred products within each of the major therapeutic categories. This list is then distributed to the plan's physicians, pharmacists, and often to the patients themselves. This type of control generally combines aspects from several of the types of formularies described above.

When a physician prescribes a medication that is not on the formulary the PBM sends an online message to the dispensing pharmacist informing him or her that the medication is nonpreferred and educating the pharmacist regarding the preferred product. The pharmacist is instructed to contact the physician and attempt to change the prescription. The MCO may pay a cognitive fee to the pharmacist for a successful intervention.

If the pharmacist is unable or does not attempt to change the prescription, the claim is adjudicated and paid. It is at this point that the PBM will intervene by asking the prescribing physician to change subsequent fills to the preferred product. If the physician agrees to the change, the plan may take one of several steps, each exhibiting varying levels of control. The plan can merely inform the patient that the physician has approved the change and request that the patient change to the preferred product when a refill is needed. While the nonpreferred product might still remain a covered benefit, the plan could change its status to nonformulary for this particular patient, or offer the nonpreferred medication at a higher copayment to the member. The pharmacy may be informed at this point, giving them ample time to contact the prescriber for a new prescription before the patient calls for a refill.

This method of control is an effective means of increasing formulary compliance. It is, however, extremely labor-intensive for the PBM and inconveniences the physician, pharmacist, and patient.

As drug classes become crowded with new products, therapeutic intervention will become more prevalent. This practice is also prompted by financial incentives in contracts between PBMs and pharmaceutical manufacturers. Additionally, HMOs must increase market share of specific products in order to justify rebates received from the manufacturers. ${ }^{5}$

\section{P\&T DECISIONS}

The process of deciding which products should be included in the formulary is a multifaceted one; however, the primary parameters by which all products are evaluated are: 1) product efficacy; 2) safety concerns; and 3) cost considerations. Because these are outpatient formularies, several other factors must be considered such as ease of use for the patient, compliance rates, dosage forms, taste, and stability. (Such factors are not important for hospital formularies where the storage and administration will be closely controlled by the nursing staff.)

Because the primary goal of any formulary system is to provide safe, appropriate, and effective prescription therapy, clinical evaluations and patient care must take precedence over all other elements. But all other factors being equal, economic considerations may be the deciding factor.

Before deciding which medications will be on the formulary, several issues must be addressed. First is the type of formulary to be implemented. Only after this has been decided can the P\&T committee begin formulating policy on drug product selection. At this point the committee can direct a subcommittee to research medications on a class-by-class basis and report back with recommendations. The committee may also choose to take advantage of the expertise of its PBM, which likely has already performed this head-to-head comparison of medications.

It is during this process of reviewing reports that the dynamic nature of a P\&T committee becomes apparent. Instead of one person taking the lead on the entire process, each person moves to the forefront when his or her area of specialty is being discussed. If the plan has an effective pharmacy director, $P \& T$ committee meetings will never become contentious. A good director knows the general restraints the committee will apply to the formulary, and will communicate this knowledge to subcommittees or individuals who are con ducting research and evaluations

Some have argued that there is no need for a $\mathrm{P} \& \mathrm{~T}$ committee to evaluate FDA-approved products. After all, manufacturers have to prove safety and efficacy before the FDA grants approval. True-however, the FDA never measures efficacy in relation to other available products. Indeed, it is this lack of head-to-head product comparison in scientific studies that causes the most complications for P\&T committees. Also, there usually is substantial postmarketing information that needs to be evaluated once the medication establishes a track record beyond the limited trials.

In addition to these decisions, there are several more issues the committee must address:

\section{Prescriptive Authority}

The P\&T committee must decide if there are to be limitations on who may prescribe medication that will be reimbursable under the drug benefit. Will the plan cover only prescriptions from physicians or can nurse practitioners and physician assistants also prescribe? Will prescriptions written by out-ofnetwork doctors be covered? If not, provisions will have to be made for processing prescriptions written by emergency room personnel.

\section{Dispensing Limits}

Decisions must be made regarding how much medication can be prescribed at one time. Most plans limit dispensing to 
a one-month supply. This is done primarily for two reasons: 1) the plan doesn't want to purchase large supplies of medication for prescriptions that might be changed or discontinued before the supply is exhausted; and 2) to prevent members from stockpiling medications.

MCOs may place dispensing limitations on conditions that are acute and self-limiting. A short supply limitation (usually less than 10 days), may also be placed on prescriptions from emergency rooms, with the rationale that the patient should see his or her primary care physician if further treatment is needed.

Most plans now offer at least one method for members to get as much as a 90-day supply of maintenance medication at one time. This is often done through mail-order pharmacies operated by the plan's PBM; such an arrangement allows the plan to provide a larger supply at a lower cost.

\section{Nonformulary Drug Use}

Before a formulary can be implemented, the P\&T committee must develop a method for authorization of nonformulary medications. During this process, protocols must be established and distributed to providers that address the following issues:

A The information necessary to process a request for a nonformulary prescription. The plan may wish to provide a standard form that is necessary to ensure that all needed information is provided. This can reduce the number of repeat requests and the amount of time required for approval. If no specific form is in place, there should be published protocols of what information is required. In most cases verbal information is too vague and is deemed inadequate. Faxed documentation is the most common form of exchange.

\ The person to whom the information is to be presented. While it may be the medical director of the plan who ultimately decides whether or not a nonformulary request will be granted, the requests are often screened in advance. This is often done by the PBM that administers the benefit for the MCO. The PBM should have guidelines on what information is required, who can provide it, and often some authority to approve or reject requests based on precisely-defined protocols.

$\Delta$ Response time. All prior authorization requests should be handled promptly since it may be crucial that therapy be initiated quickly for the well-being of the patient. If a decision cannot be reached due to lack of information or some other extenuating circumstance, there should be a method for the pharmacist to accept full payment, with patient reimbursement to occur once a decision has been reached.

\section{Additions to the Formulary}

A formulary must be an ever-changing list of drugs. New medications are always being approved, older ones lose their patent protection or obtain new indications, and new clinical information comes to light regarding both safety and efficacy. In addition to these clinical concerns, the economic landscape is constantly changing. New manufacturer contracts can restore coverage of products that had previously been restricted. The P\&T committee must constantly review its formulary and establish a procedure for providers to request the addition of medication to the formulary. There should be a form that providers may complete requesting changes. The completed form should indicate the medication requested, what it would replace, and its advantages.

These requests should be presented to the plan's medical director, pharmacy director, and head of the P\&T committee for discussion at the next shceduled meeting.

\section{EDUCATION}

The success of any system depends largely on the attention paid by the P\&T committee to the educational component of the formulary. This education needs to occur at three levels: physician, pharmacist, and patient. If any of these are missed there will surely be a breakdown in formulary compliance.

One aspect often overlooked is the use of the formulary as an educational tool and a method by which to stay current with the growing number of drugs in the therapeutic armamentarium. ${ }^{6}$

For these reasons, the formulary should have an information component explaining the rationale behind decisions. This may be in the form of drug monographs published with the formulary, periodic newsletters to providers, and/or in-service educational programs for medical personnel. The goal of formulary education is to furnish providers with the information needed to provide a high standard of care based on a rational decision-making process.

Educating the pharmacist is paramount because in many formulary systems the pharmacist performs the 'gatekeeper' role with respect to medications. Keeping the pharmacist informed regarding any changes in formulary policy or content, along with the rationale behind the changes will lead to greater formulary compliance. The community pharmacist often performs the task of explaining the formulary structure and purpose to the actual member, so it is imperative to have accurate and current information at hand.

Many plans now offer financial incentives to pharmacists for their participation in formulary compliance. However, to ensure the success of such incentive plans, it's imperative that the pharmacist is clear on what benefits he or she will receive, and is convinced that the additional time required is outweighed by the potential gain.

Patient acceptance and encouragement of formulary compliance is extremely desirable but cannot be accomplished without patient education. The MCO needs to educate the patient regarding the economic and medical rationale behind their formulary system. Patient education efforts should not 
a one-month supply. This is done primarily for two reasons 1) the plan doesn't want to purchase large supplies of medication for prescriptions that might be changed or discontinued before the supply is exhausted; and 2) to prevent members from stockpiling medications.

MCOs may place dispensing limitations on conditions that are acute and self-limiting. A short supply limitation (usually less than 10 days), may also be placed on prescriptions from emergency rooms, with the rationale that the patient should see his or her primary care physician if further treatment is needed.

Most plans now offer at least one method for members to get as much as a 90-day supply of maintenance medication at one time. This is often done through mail-order pharmacies operated by the plan's PBM; such an arrangement allows the plan to provide a larger supply at a lower cost.

\section{Nonformulary Drug Use}

Before a formulary can be implemented, the P\&T committee must develop a method for authorization of nonformulary medications. During this process, protocols must be established and distributed to providers that address the following issues:

A The information necessary to process a request for a nonformulary prescription. The plan may wish to provide a standard form that is necessary to ensure that all needed information is provided. This can reduce the number of repeat requests and the amount of time required for approval. If no specific form is in place, there should be published protocols of what information is required. In most cases verbal information is too vague and is deemed inadequate. Faxed documentation is the most common form of exchange.

A The person to whom the information is to be presented. While it may be the medical director of the plan who ultimately decides whether or not a nonformulary request will be granted, the requests are often screened in advance. This is often done by the PBM that administers the benefit for the MCO. The PBM should have guidelines on what information is required, who can provide it, and often some authority to approve or reject requests based on precisely-defined protocols.

A Response time. All prior authorization requests should be handled promptly since it may be crucial that therapy be initiated quickly for the well-being of the patient. If a decision cannot be reached due to lack of information or some other extenuating circumstance, there should be a method for the pharmacist to accept full payment, with patient reimbursement to occur once a decision has been reached.

\section{Additions to the Formulary}

A formulary must be an ever-changing list of drugs. New medications are always being approved, older ones lose their patent protection or obtain new indications, and new clinical information comes to light regarding both safety and efficacy. In addition to these clinical concerns, the economic landscape is constantly changing. New manufacturer contracts can restore coverage of products that had previously been restricted. The P\&T committee must constantly review its formulary and establish a procedure for providers to request the addition of medication to the formulary. There should be a form that providers may complete requesting changes. The completed form should indicate the medication requested, what it would replace, and its advantages.

These requests should be presented to the plan's medical director, pharmacy director, and head of the P\&T committee for discussion at the next shceduled meeting.

\section{EDUCATION}

The success of any system depends largely on the attention paid by the P\&T committee to the educational component of the formulary. This education needs to occur at three levels: physician, pharmacist, and patient. If any of these are missed there will surely be a breakdown in formulary compliance.

One aspect often overlooked is the use of the formulary as an educational tool and a method by which to stay current with the growing number of drugs in the therapeutic armamentarium. ${ }^{6}$

For these reasons, the formulary should have an information component explaining the rationale behind decisions. This may be in the form of drug monographs published with the formulary, periodic newsletters to providers, and/or in-service educational programs for medical personnel. The goal of formulary education is to furnish providers with the information needed to provide a high standard of care based on a rational decision-making process.

Educating the pharmacist is paramount because in many formulary systems the pharmacist performs the 'gatekeeper' role with respect to medications. Keeping the pharmacist informed regarding any changes in formulary policy or content, along with the rationale behind the changes will lead to greater formulary compliance. The community pharmacist often performs the task of explaining the formulary structure and purpose to the actual member, so it is imperative to have accurate and current information at hand.

Many plans now offer financial incentives to pharmacists for their participation in formulary compliance. However, to ensure the success of such incentive plans, it's imperative that the pharmacist is clear on what benefits he or she will receive, and is convinced that the additional time required is outweighed by the potential gain.

Patient acceptance and encouragement of formulary compliance is extremely desirable but cannot be accomplished without patient education. The MCO needs to educate the patient regarding the economic and medical rationale behind their formulary system. Patient education efforts should not 
a one-month supply. This is done primarily for two reasons: 1) the plan doesn't want to purchase large supplies of medication for prescriptions that might be changed or discontinued before the supply is exhausted; and 2) to prevent members from stockpiling medications.

MCOs may place dispensing limitations on conditions that are acute and self-limiting. A short supply limitation (usually less than 10 days), may also be placed on prescriptions from emergency rooms, with the rationale that the patient should see his or her primary care physician if further treatment is needed.

Most plans now offer at least one method for members to get as much as a 90-day supply of maintenance medication at one time. This is often done through mail-order pharmacies operated by the plan's PBM; such an arrangement allows the plan to provide a larger supply at a lower cost.

\section{Nonformulary Drug Use}

Before a formulary can be implemented, the P\&T committee must develop a method for authorization of nonformulary medications. During this process, protocols must be established and distributed to providers that address the following issues:

- The information necessary to process a request for a nonformulary prescription. The plan may wish to provide a standard form that is necessary to ensure that all needed information is provided. This can reduce the number of repeat requests and the amount of time required for approval. If no specific form is in place, there should be published protocols of what information is required. In most cases verbal information is too vague and is deemed inadequate. Faxed documentation is the most common form of exchange.

A The person to whom the information is to be presented. While it may be the medical director of the plan who ultimately decides whether or not a nonformulary request will be granted, the requests are often screened in advance. This is often done by the PBM that administers the benefit for the MCO. The PBM should have guidelines on what information is required, who can provide it, and often some authority to approve or reject requests based on precisely-defined protocols.

- Response time. All prior authorization requests should be handled promptly since it may be crucial that therapy be initiated quickly for the well-being of the patient. If a decision cannot be reached due to lack of information or some other extenuating circumstance, there should be a method for the pharmacist to accept full payment, with patient reimbursement to occur once a decision has been reached.

\section{Additions to the Formulary}

A formulary must be an ever-changing list of drugs. New medications are always being approved, older ones lose their patent protection or obtain new indications, and new clinical information comes to light regarding both safety and efficacy. In addition to these clinical concerns, the economic landscape is constantly changing. New manufacturer contracts can restore coverage of products that had previously been restricted The P\&T committee must constantly review its formulary and establish a procedure for providers to request the addition of medication to the formulary. There should be a form that providers may complete requesting changes. The completed form should indicate the medication requested, what it would replace, and its advantages.

These requests should be presented to the plan's medical director, pharmacy director, and head of the P\&T committee for discussion at the next shceduled meeting.

\section{EDUCATION}

The success of any system depends largely on the attention paid by the $P \& T$ committee to the educational component of the formulary. This education needs to occur at three levels: physician, pharmacist, and patient. If any of these are missed there will surely be a breakdown in formulary compliance.

One aspect often overlooked is the use of the formulary as an educational tool and a method by which to stay current with the growing number of drugs in the therapeutic armamentarium. ${ }^{6}$

For these reasons, the formulary should have an information component explaining the rationale behind decisions. This may be in the form of drug monographs published with the formulary, periodic newsletters to providers, and/or in-service educational programs for medical personnel. The goal of formulary education is to furnish providers with the information needed to provide a high standard of care based on a rational decision-making process.

Educating the pharmacist is paramount because in many formulary systems the pharmacist performs the 'gatekeeper' role with respect to medications. Keeping the pharmacist informed regarding any changes in formulary policy or content, along with the rationale behind the changes will lead to greater formulary compliance. The community pharmacist often performs the task of explaining the formulary structure and purpose to the actual member, so it is imperative to have accurate and current information at hand.

Many plans now offer financial incentives to pharmacists for their participation in formulary compliance. However, to ensure the success of such incentive plans, it's imperative that the pharmacist is clear on what benefits he or she will receive, and is convinced that the additional time required is outweighed by the potential gain.

Patient acceptance and encouragement of formulary compliance is extremely desirable but cannot be accomplished without patient education. The MCO needs to educate the patient regarding the economic and medical rationale behind their formulary system. Patient education efforts should not 
Table 1. Awareness of Formulary Among Consumers ${ }^{5}$

\begin{tabular}{lccc}
\hline Type of Insurance & Yes & No & Do Not Know \\
\hline Managed Care & $18.8 \%$ & $54.2 \%$ & $27.0 \%$ \\
\hline Indemnity & $8.5 \%$ & $75.0 \%$ & $16.5 \%$ \\
\hline Medicare & $14.0 \%$ & $57.7 \%$ & $28.3 \%$ \\
\hline Medicaid & $49.0 \%$ & $27.1 \%$ & $24.0 \%$ \\
\hline CHAMPUS & $15.1 \%$ & $60.3 \%$ & $24.7 \%$ \\
\hline
\end{tabular}

Note: Totals do not equal $100 \%$ due to rounding

only explain the system and how decisions are made, they must also explain the role of the patient and the importance of formulary compliance to both the patient and the plan. As Table 1 shows, the majority of consumers are not aware that their plans have formulary restrictions.

If a health plan fully educates members regarding the formulary and its purpose, it can expect members to request the use of formulary medication more often, especially if there are economic incentives for the member. Education will also enhance perceived quality of care should the member experience a delay-due to a nonformulary medication.

\section{DRUG USE EVALUATION}

An adjunct to the formulary system is a drug utilization evaluation (DUE) program. This may be overseen by the P\&T committee, but will often be an independent committee.

The purpose of DUE is to continuously evaluate the effectiveness, safety, and appropriate use of drugs. ${ }^{7}$ The properlydesigned DUE program will have specific criteria to measure the appropriateness of drug use and the over/underutilization of drug therapy. It will look at both the usage and the outcomes to determine if medication is being properly utilized. The DUE process should develop and present treatment guidelines and drug use criteria. The outcomes can then be evaluated in patients who followed these guidelines and those who followed other treatment pathways. Comparisons among both groups will allow rational evaluation of the formulary and the outcomes it is producing.

Because of rapid changes in medical technology and drug therapy choices, a formulary must constantly be reevaluated and maintained. DUE is a valuable mechanism for evaluating the effectiveness of the formulary. Table 2 presents the key elements of a comprehensive DUE.

\section{OUTCOMES-BASED FORMULARIES}

Until recently, the clinical review of medications to determine which medications should be considered for inclusion on a formulary has been largely based on the random clinical trials (RCTs) performed for FDA approval. For many years
Table 2. Elements of a Comprehensive DUE Program ${ }^{1}$

\section{Appropriateness of Drug Use}

$\boldsymbol{\Delta}$ Indications

$\Delta$ Contraindications

A Risk factors

A Age

- Gender

A Concurrent drug use (drug interactions)

\section{Overutilization/Underutilization}

^ Dosage

A Quality of dose

A Frequency of dose

A Interval between prescriptions

A Duration of therapy

these RCTs have been the gold standard used to judge all drug treatments. We are now seeing increased reliance on another method to judge which agent is the most appropriate. This new standard is patient outcomes. Pharmacoepidemiology looks only at whether a specific intervention does what it was intended to do for a specific population. Treatments are now being evaluated based on pharmacoeconomics, which encompasses an assessment of effectiveness as well as cost.

Outcomes-based formularies look at pharmacoeconomics and also factor in quality-of-life issues. We are starting to see a discernible difference between effectiveness and efficacy. Outcomes look at the overall impact of treatment to determine the effectiveness of a treatment in clinical, social, and economic terms rather than the previous efficacy and cost.

The growing importance of Healthcare Employer Data and Information Set (HEDIS) and the National Committee for Quality Assurance evaluations of managed care plans is causing a greater awareness of outcomes data. As a standard is developed to compare HMOs based on this outcomes data, it is likely we will see outcomes-based formularies that encompass the entire cost of care as opposed to just the cost of the medication.

There is also a tremendous focus now on disease state management (DSM). This is a global approach to treatment that seeks to encompass all aspects of treating a specific disease. From a medication standpoint this includes, but is not limited to, pharmacology, drug selection, contraindications, risk factors, adverse effects, drug interactions, dosing, and patient education and counseling.

DSM will develop treatment pathways that may become the criteria for treatment options. It's unlikely that these will be the only treatment methods allowed, but it may require documentation from the physician to prescribe outside these pathways. Instead of a blanket listing applied to an MCO's entire membership, these guidelines (and thus the formulary) would become diagnosis driven. 
Table 1. Awareness of Formulary Among Consumers ${ }^{6}$

\begin{tabular}{lccc}
\hline Type of Insurance & Yes & No & Do Not Know \\
\hline Managed Care & $18.8 \%$ & $54.2 \%$ & $27.0 \%$ \\
\hline Indemnity & $8.5 \%$ & $75.0 \%$ & $16.5 \%$ \\
\hline Medicare & $14.0 \%$ & $57.7 \%$ & $28.3 \%$ \\
\hline Medicaid & $49.0 \%$ & $27.1 \%$ & $24.0 \%$ \\
\hline CHAMPUS & $15.1 \%$ & $60.3 \%$ & $24.7 \%$ \\
\hline
\end{tabular}

Note: Totals do not equal $100 \%$ due to rounding

only explain the system and how decisions are made, they must also explain the role of the patient and the importance of formulary compliance to both the patient and the plan. As Table 1 shows, the majority of consumers are not aware that their plans have formulary restrictions.

If a health plan fully educates members regarding the formulary and its purpose, it can expect members to request the use of formulary medication more often, especially if there are economic incentives for the member. Education will also enhance perceived quality of care should the member experience a delay due to a nonformulary medication.

\section{DRUG USE EVALUATION}

An adjunct to the formulary system is a drug utilization evaluation (DUE) program. This may be overseen by the P\&T committee, but will often be an independent committee.

The purpose of DUE is to continuously evaluate the effectiveness, safety, and appropriate use of drugs. ${ }^{7}$ The properlydesigned DUE program will have specific criteria to measure the appropriateness of drug use and the over/underutilization of drug therapy. It will look at both the usage and the outcomes to determine if medication is being properly utilized. The DUE process should develop and present treatment guidelines and drug use criteria. The outcomes can then be evaluated in patients who followed these guidelines and those who followed other treatment pathways. Comparisons among both groups will allow rational evaluation of the formulary and the outcomes it is producing.

Because of rapid changes in medical technology and drug therapy choices, a formulary must constantly be reevaluated and maintained. DUE is a valuable mechanism for evaluating the effectiveness of the formulary. Table 2 presents the key elements of a comprehensive DUE.

\section{OUTCOMES-BASED FORMULARIES}

Until recently, the clinical review of medications to determine which medications should be considered for inclusion on a formulary has been largely based on the random clinical trials (RCTs) performed for FDA approval. For many years
Table 2. Elements of a Comprehensive DUE Program ${ }^{1}$

\section{Appropriateness of Drug Use}

A Indications

A Contraindications

$\Delta$ Risk factors

- Age

- Gender

A Concurrent drug use (drug interactions)

\section{Overutilization/Underutilization}

^ Dosage

$\Delta$ Quality of dose

$\Delta$ Frequency of dose

$\Delta$ Interval between prescriptions

- Duration of therapy

these RCTs have been the gold standard used to judge all drug treatments. We are now seeing increased reliance on another method to judge which agent is the most appropriate. This new standard is patient outcomes. Pharmacoepidemiology looks only at whether a specific intervention does what it was intended to do for a specific population. Treatments are now being evaluated based on pharmacoeconomics, which encompasses an assessment of effectiveness as well as cost.

Outcomes-based formularies look at pharmacoeconomics and also factor in quality-of-life issues. We are starting to see a discernible difference between effectiveness and efficacy. Outcomes look at the overall impact of treatment to determine the effectiveness of a treatment in clinical, social, and economic terms rather than the previous efficacy and cost.

The growing importance of Healthcare Employer Data and Information Set (HEDIS) and the National Committee for Quality Assurance evaluations of managed care plans is causing a greater awareness of outcomes data. As a standard is developed to compare HMOs based on this outcomes data, it is likely we will see outcomes-based formularies that encompass the entire cost of care as opposed to just the cost of the medication.

There is also a tremendous focus now on disease state management (DSM). This is a global approach to treatment that seeks to encompass all aspects of treating a specific disease. From a medication standpoint this includes, but is not limited to, pharmacology, drug selection, contraindications, risk factors, adverse effects, drug interactions, dosing, and patient education and counseling.

DSM will develop treatment pathways that may become the criteria for treatment options. It's unlikely that these will be the only treatment methods allowed, but it may require documentation from the physician to prescribe outside these pathways. Instead of a blanket listing applied to an MCO's entire membership, these guidelines (and thus the formulary) would become diagnosis driven. 


\section{CRITICISM}

The formulary system has been a part of the American health care environment for decades and has been increasing in magnitude in recent years. However, it is not without its critics. A study published in the March 1996 issue of the American Journal of Managed Care ${ }^{8}$ is often cited by critics of managed care formularies. The article suggested, after studying six HMOs, that restrictive formularies may result in decreased quality of patient care, higher use of health services, and greater overall cost of medical care.

The study posits statistical correlations only and does not link utilization with patient disease states, nor does it address quality of care or look at the efficacy of drugs included in the formularies. ${ }^{9}$ The study was flawed in that it failed to collect information prior to implementation of the formularies in these plans to use as a baseline for comparison. It does, however, raise some interesting questions regarding the effect of formularies on medical cost. While it is by no means definitive, it demonstrates the need to constantly monitor and adjust the formulary system. The more outcomes-based data that is obtained, the sooner we'll have answers to many of the questions raised by this study.

The other main focus of criticism directed at formularies of late has been in the area of therapeutic substitution. The point of most of this criticism has been that formularies limit the choices physicians have to treat patients and, in some cases, have created potential conflicts of interest.

\section{Judicial Threats}

In 1995 Minnesota Attorney General Hubert H. Humphrey III led a 17 -state enforcement action against a large PBM. The company paid a $\$ 1.9$ million fine to settle the charges and agreed to change its practices regarding therapeutic substitution. ${ }^{10}$ The company had just recently undergone a merger and thus it was in the position of promoting the products of its parent company. The PBM agreed to inform doctors when it contacted them that the preferred drug was manufactured by its parent company. This type of conflict of interest will undoubtedly remain a problem for those PBMs that have been vertically integrated with pharmaceutical manufacturers.

\section{Governmental Reports}

A report issued in 1996 by New York City's Public Advocate (a citywide elected post) was extremely critical of the practice of therapeutic substitution. The report claimed that the practice was limiting the public's access to valuable drugs: "We found that commercial rather than public health concerns are driving this remarkable transformation of the medical marketplace." ${ }^{\prime 11}$ Among the recommendations in his report are:

A Pharmacists should be required to inform patients of all switches.
Table 3. Measures Employers are Likely to Pursue ${ }^{13}$

\section{Likelihood of employers using a prescription drug formulary over the next two years}

$\begin{array}{ll}\text { Very Likely } & 29 \% \\ \text { Somewhat Likely } & 38 \% \\ \text { Somewhat Unlikely } & 21 \% \\ \text { Very Unlikely } & 11 \% \\ \text { Undecided } & 1 \%\end{array}$

Likelihood of employers using a managed prescription plan to reduce medical costs and measure outcomes over the next two years

$\begin{array}{ll}\text { Very Likely } & 35 \% \\ \text { Somewhat Likely } & 42 \% \\ \text { Somewhat Unlikely } & 9 \% \\ \text { Very Unlikely } & 7 \% \\ \text { Undecided } & 7 \%\end{array}$

A Health insurance plans which are already required to disclose their formularies on request should also reveal their preferred drugs.

A Pharmacists should refrain from therapeutic substitutions in most cases, unless the substituted prescription is bioequivalent.

A Pharmaceutical manufacturers should divest themselves of PBMs.

\section{Legislative Threat}

On January 20, 1997 legislation was introduced in the Virginia House of Representatives taking aim at the practice of therapeutic substitution. The Anti-Drug Switching Patient Protection Act (HB2714) stipulated that "no person shall solicit or encourage the prescribing practitioner...to substitute a prescription drug... with any chemically dissimilar prescription drug" and that pharmacists who knew that the prescriber had been solicited with money to switch chemically dissimilar drugs could not dispense the drugs. The bill imposes fines ranging from $\$ 10$ to $\$ 5,000$ plus attorneys' fees and costs. ${ }^{12}$ The bill was referred to committee, where by a 13-7 vote it was tabled and another committee was set up to study the issue.

A bill was recently passed by the Missouri legislature that would require the approval of both physician and patient before any therapeutic substitution could occur.

The heightened attention spotlighting the practice of therapeutic substitution, as well as the criticisms raised regarding formularies in general is not reason enough to abandon these successful managed care techniques. On the contrary, such criticism motivates us to constantly reevaluate the formulary to be sure that the patient's interest is being served. 


\section{THE FUTURE}

In 1995, William M. Mercer, Inc., a New York City-based employee-benefits compensation and human resource management and consulting firm, conducted the "1.995 National Survey of Advanced Pharmacy Benefit Management."13 Among other findings, the survey determined a number of measures that employers are likely to pursue (See Table 3).

These trends indicate the value that employers place on using formularies to managed escalating prescription costs Any attempts to limit the use of the less-restrictive formularycontrol mechanisms, such as therapeutic substitution, may cause the implementation of stricter closed formularies. With the privatization of Medicaid and Medicare, managed care organizations are going to get less money (for patients' drug therapy) than they are getting from employer groups. There are going to be strong incentives to put more restrictive formularies in place for those kinds of patients. ${ }^{14}$

\section{CONCLUSION}

While certain aspects of the formulary system may need to be revisited, as some studies have suggested, there is no doubt that formularies are an effective pharmaceutical care instrument. In its position statement on formularies the Academy of Managed Care Pharmacy states that, "AMCP supports a well-designed and properly-administered formulary to assist in effectively managing a patient's total medical care regimen. A formulary enhances quality of care by encouraging the use of those prescription medications that are demonstrated to be safe, effective, and produce superior patient outcomes. ${ }^{15}$ Formularies are certain to have an increasing impact on the practice of pharmacy. Managed care providers must completely understand the entire formulary system, its development, and its future, in order to fully interact with the system and also play a role in assuring optimal patient outcomes within the managed care environment.

\section{References}

1) Medical Interface's Facts \& Figures, Bronxville, New York, Medicom International 1996.

2) Ito SM, Blackburn $S$, eds. A Pharmacist Guide to Principles and Practices of Managed Care. FMCP 1995.

3) Anonymous. Task Force on Prescription Drugs: Final Report. Washington, DC: US Department of Health Education and Welfare; 1969: 40

4) Shepherd MD, Salzman RD. The Formulary

Decision-Making Process in a Health Maintenance Organization Setting. PharmacoEconomics 1994; 5(1): 29-38.
5) Strand LM, Cipolle RJ, Marley PC. Pharmaceutical Care: An Introduction. Current Concepts. Kalamazoo, Mi; The Upjohn Co, 1992.

6) Navarro RP. 1996 Trends \& Forecasts.

CibaGeneva Pharmacy Benefit Report.

7) Chitwood KK, Edgren B, Schultz N. Drug

Formularies. Medical Interface 1993; 6(12): 72-75.

8) Horn SD, Sharkey PD, Tracy DM, et al.

Intended and Unintended Consequences of $\mathrm{HMO}$

Cost Containment Strategies: Results from the

Managed Care Outcomies Project. Am J Managed

Care 1996; 2: 253-64.

9) Cathey JT. The End of the Restrictive

Formulary? Drug Benefit Trends 1996: 8 (4)
10) Anonymous. 1995 The Year in Review. Drug Store New For The Pharmacist 1996; 6(4). 11) Conlin MF. NY Report Decries Rx Drug Switching by PBMs. Drug Topics 1997; 141(2): 14-16.

12) Ukens, C. Sub Attack Virginia takes aim at drug switching. Drug Topics 1997; 141(6): 21. 13) Anonymous. Future Initiatives in Managed Care Pharmacy: Employer Survey. Drug Benefit Trends 1996; 8(6): 8-9.

14) Muirhead G. Closing Formularies No Easy, Overnight Job. Drug Topics 1996; 140 (3): 59.

15) Position Statement on Formularies. Academy of Managed Care Pharmacy; February 1997. 